\title{
Anticipatory deficits in psychosis during social cognition task revealed by task-based EEG dynamic functional connectivity analysis
}

\author{
Anna Padée*, Pascal Missonnier*, Anne Prévot, \\ Grégoire Favre, Isabelle Gothuey, Jonas Richiardi
}

\begin{abstract}
Introduction Schizophrenia affects a wide range of cognitive functions. These deficits present during socioeconomic game play, such as the Ultimatum Game (UG), which combines decision-making with social interaction: participants make offers of money (proposer) or decide whether to accept offers or not (responder). Correct activation of neuronal bases has been reported in schizophrenic patients following a proposition of money, while the same networks were not activated in response to the offered amount of money. It is unclear, however, if abnormalities in the neural networks' commitment supporting the anticipation of the stimulus for final social decision-making may explain this discrepancy between both experimental conditions.

Method We compared schizophrenia patients $(\mathrm{N}=19)$ with healthy controls $(\mathrm{N}=24)$ during the UG task, computing EEG imaginary part of coherency (ImCoh). Fronto-occipital and temporal interhemispheric ImCoh in alpha $(8-13 \mathrm{~Hz})$ and beta $(14-30 \mathrm{~Hz})$ frequency bands was estimated to assess the strength of functional connectivity between these brain regions.

Results In the responder condition, we found significant group differences in alpha band between frontal and occipital electrodes, starting $0.7 \mathrm{~s}$ before stimulus onset $(\mathrm{p}=0.043$, corrected for multiple comparisons). In contrast, there was no significant group difference in the beta band. In the proposer condition, no difference in connectivity was found in either frequency band.

Conclusion These findings support the idea of a psychosis-related dysfunction of fronto-occipital cortical networks engaged in the anticipation of the proposition during the decision part of the game. In contrast, patients with schizophrenia may have preserved functional connectivity in the proposer condition, indicating that the timing of anticipation commitment plays a key role in adapted activation of neural bases related to human interaction in schizophrenia.
\end{abstract}

\section{Introduction}

Schizophrenia is a severe psychiatric illness characterized by a highly variable set of symptoms [1], including impairments of interaction with other individuals known as social cognition [2]. These may appear as early as the prodromal phase and can predict illness onset $[3,4]$. Therefore, it is crucial to identify the underlying neural mechanisms of these specific deficits, especially considering that social cognition may be used to guide treatment aimed at improving the integration of these patients in society.

The evaluation of social decision-making processes corresponds to mental state attribution capacity, also called theory of mind (ToM), or mentalizing. It allows the maintenance of interactions in a social environment and requires performing interactive tasks. The socioeconomic Ultimatum Game (UG) appears to be particularly suitable to study these social interactions $[5,6]$. In this game one player (the proposer) has a certain amount of money, of which he offers a share to the second player (the responder). Although it would be statistically optimal to propose the smaller sum of money, and respond by accepting any offer [7], proposers typically offer $40 \%$ of the money to be shared and responders reject $50 \%$ of the offers smaller than $30 \%$ of the money proposed [8]. This deviation from "rational" strategies highlights that behaviors incorporate equity, supporting the view that the UG gives the possibility to evaluate social decision-making ecologically [9].

The rare studies using the UG conducted in patients with schizophrenia revealed that these patients make more fair offers as proposers and accept more unfair offers as responders, and also make more erratic offers after a rejection [10-12]. These findings demonstrate a striking difficulty to adapt their strategy and anticipate their counterparts' intentions in an environment of rapid social interaction. Consistent with these observations, we reported in an earlier contribution a decreased amplitude of the feedback-related negativity (FRN) component, an event-related potential (ERP) component within 320-360 ms after stimulus onset, suggesting difficulties of patients with schizophrenia in interpreting other's behavior. In contrast, no difference reflecting social decision-making linked to the disease was found in the proposer condition, supporting the idea of different involvement of the ToM between the UG conditions. Interestingly, functional imaging studies revealed that neural activations vary over time during the ToM tasks in patients with schizophrenia [13]. This promotes the scenario of a poorly coordinated activation of neural networks involved in anticipating a partner's behavior and elaborating his responses during social interaction in the responder condition in these patients. Eventmodulated electroencephalographic (EEG) dynamic analysis in frequency bands of interest remains the most appropriate method to explore pathological functioning in this context. 
Physiologically, synchronous neural oscillatory activity between different subsets of brain regions [14] or phase synchronization is a fundamental mechanism of brain communication. In fact, cerebral oscillations set up functional networks supporting many cognitive functions by transiently triggering and suppressing oscillatory synchronization in a wide variety of frequencies (i.e., coherence) $[15,16]$. In controls, numerous previous reports pointed to an increase of the alpha dominant band (8-12 Hz) during social interaction, indicating the ability to anticipate the other player's behavior $[9,17]$. Similar results were observed during social interactions with alpha power increasing in relation to anticipation of other's player behavior. More interestingly, a recent connectivity study pointed to a correlation between the power of alpha desynchronization over fronto-occipital areas and the level of expertise of the players in the task [17], supporting the idea of a functional relationship between anticipation and accuracy of judgments. Furthermore, a recent connectivity study during the UG task revealed that individual acceptance rates were highly correlated with a fronto-occipital functional network formation coordinated by alpha-band [18]. Finally, mental simulation directed to other's preference also modulates oscillations at higher frequencies within the beta band $(15-25 \mathrm{~Hz})$ [19]. Recently, a study showed that the power of beta oscillations over the right temporoparietal area (rTPJ) was correlated with an individual's ability to predict the preference of others during an inference task [19], implying that the strength of beta synchronization over these areas might indicate a certain level of anticipation. In line with this finding, similar results were reported during top-down attention engagement [20, 21]. Importantly, subtle abnormalities of the temporal regions have long been identified in schizophrenia [22], which may contribute to the social cognition deficits encountered by these patients.

Other work documented a lower alpha power in frontal and temporoparietal regions correlated with more risky offers made when patients with chronic schizophrenia anticipate responses in the proposer condition of the UG [22]; that work did not investigate network dynamics or the responder condition. We previously reported a decrease of amplitude of an ERP component related to decision-making in patients with schizophrenia in the responder, but not in the proposer condition in the UG paradigm [23]. Thus, Whether these changes are preceded by a disruption of functional connectivity is still not clearly identified. To address this issue, we performed an analysis of event-related imaginary part of coherency ( $\mathrm{mCOh}$ ) between fronto-occipital areas in the alpha band before and after offer onset in both UG conditions, as well as event-related ImCoh between intertemporal areas in the beta band in healthy controls and patients with chronic schizophrenia. We hypothesized that fronto-occipital aberrant connectivity in alpha band may occur before the offer is made in the responder condition in patients with schizophrenia, which is the time period to infer social judgments for subsequent decision making.

\section{Material and Methods}

\subsection{Participants}

The present work is based on data collected for a previous study performed in a cohort of healthy controls and patients with schizophrenia [23], which is re-processed, newly analysed, and made open access with the present work (see Data Availability section). The protocol was approved by the Ethics Committee of the University of Fribourg, Switzerland. Briefly, twenty-four healthy controls (HC) and nineteen patients with schizophrenia (SCZ) were recruited. SCZ patients were hospitalized at the Mental Health Network Fribourg (RFSM) for a psychotic decompensation. An experienced independent psychiatrist made the diagnosis according to the International Statistical Classification of Diseases and Related Health Problems [24]. Fourteen patients were diagnosed with paranoid schizophrenia (F20.0) and five with acute and transient psychotic disorders (F23). Patients with drug use, as well as with history of sustained head injury or other neurologic or psychiatric disorders, were excluded. The severity of the patients' symptoms was rated using items of the Brief Psychiatric Rating Scale (BPRS) [23, 25]. Controls had no history of sustained head injury or other neurologic or psychiatric disorders. All participants had normal or corrected-to-normal visual acuity and none of them suffered from any severe physical impairment. Demographic characteristics of all participants and clinical information of patients are provided in Table 2.

\subsection{Task and procedure}

Participants performed an adapted version of the UG, in which the player acting as "proposer" has a fixed sum of money (10 Swiss francs $=10 \mathrm{CHF}$ ) available and has to propose a share of this money to the one acting as "responder", who can either accept or reject this offer. Money is shared in the proposed ratio between both players if the responder accepts the proposal, but both end up with nothing if he refuses. Participants were playing against a computer player, but they were not told explicitly (task instructions mentioned a "second player"). As responder, the computer program was implemented to simulate a human strategy, with increasing acceptance rates for higher offers. Previous studies [26, 27] reported that participants reject unfair offers made by humans at a higher rate than those made by computer players (see Fig. 1 and [23] for thorough description of the experimental procedures). The overall experiment lasted about $70 \mathrm{~min}$. 


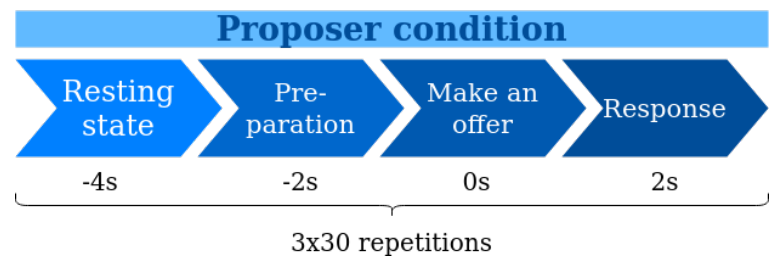

(a) Experimental paradigm in the proposer condition

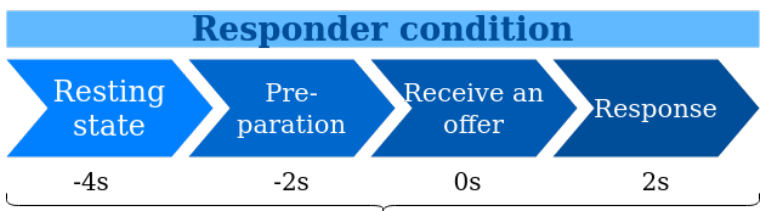

$3 \times 30$ repetitions

(b) Experimental paradigm in the responder condition

Figure 1: Illustration of the Ultimatum Game. In the present version of the UG, each participant played both the role of the proposer (90 trials) and of the responder (90 trials) in three alternate blocks of 30 trials each (180s per block). Before each block, participants were informed about the nature of the task. Each trial started with a preparatory period of 2 seconds, where participants maintained their gaze on a central fixation cross on the screen (Preparation). Next, a message appeared to make a proposition ("Please, make your offer"; A Proposer) or respond to an offer ("Do you accept or reject the offer of $[1, \ldots$. , 9] CHF?"; B - Responder). This time point was considered as $t=0$ for the EEG analyses. The response to the offer was displayed simultaneously when the participants pressed the button indicating their decision and presented as a smiling face for "Accept" or a frowning face for "Reject" in the proposer condition, whereas the face remained neutral to minimize the influence of positive or negative facial expressions in the responder condition. Participants were also told to play the UG trying to maximize their gain as much as possible.

\subsection{Electrophysiological recordings}

Continuous EEG was recorded using 128 active surface $\mathrm{Ag} / \mathrm{AgCl}$ electrodes (ActiveTwo MARK II Biosemi EEG System, BioSemi B.V., Amsterdam, Netherlands) mounted on a head cap (NeuroSpec Quick Cap) and referenced to the common mode sense (CMS; active electrode). Linked right and left mastoid electrodes were used for a later re-referencing process. Additionally, right-, left-, supra-, and infra-orbital electrodes monitored horizontal and vertical eye movements. Electrode impedances were kept below $20 \mathrm{k} \Omega$. Electrophysiological signals were sampled at $2048 \mathrm{~Hz}$ (DC amplifiers and software by Biosemi, USA). Markers corresponding to stimuli presentations and responses (proposer and responder offer types) were automatically documented with markers in the continuous EEG file, which were used off-line to segment the continuous EEG data into timelocked epochs.

\subsection{Electrophysiological data processing}

The EEG trials were automatically scanned for contamination by muscular or electrode artifacts (criteria for rejection: voltage step $>50 \mu \mathrm{V} / \mathrm{ms}$ or peak-to peak deflection within 300 -ms intervals $>200 \mu \mathrm{V} / \mathrm{ms}$ ), and the remaining trials were inspected visually to control for residual minor artifacts. After rejecting bad trials, there were on average $86.8 \pm 3.6(\mathrm{HC})$ and $83.8 \pm 4.8(\mathrm{SCZ})$ trials left of 90 per participant. Bad channels were identified visually and interpolated from the surrounding electrodes by spherical splines. Raw EEG data were re-referenced to the average reference offline. This first stage of the EEG analysis was conducted with Brain Vision Analyzer 2 software (Brain Products GmbH). EEG signals were corrected for blinks and eye movement artifacts through an ICA $[28,29]$, which parameters were carefully selected for each individual to minimize any residual effects on the visually-inspected EEG signal. The data were low-pass filtered with a FIR filter with the cutoff frequency of $40 \mathrm{~Hz}(-6 \mathrm{~dB}$ cutoff frequency: $45 \mathrm{~Hz})$. The total analysis window was 4 seconds, starting 2 seconds before spacebar press (Figure 1).

In extra-cranial EEG, solving the problem of so-called volume conduction effects is a challenge in the analysis of cerebral connectivity. Specifically, volume conduction reflects the diffusion of a single cortical source over multiple electrodes, possibly resulting in spurious connectivity measures. To address this effect, we used a spatial filter approach technique to improve the spatial resolution of the EEG and connectivity analysis based on imaginary part of coherency (see below, point 2.5). EEG signals were filtered by surface Laplacian calculation (regularized 3-D spline function, order 4) which acts as a high-pass spatial filter that reduces head volume conduction and cancels out reference electrode influence [30-33]. Therefore, it provides EEG topographies with more defined peaks than those of scalp potential [34-36]. The spherical spline surface Laplacian from the MNE package [37] was used to compute the transform, with the parameters of $\lambda^{2}=10^{-3}$ and stiffness $=3$. Effects of varying these parameters in our data are shown in Supplementary Material, Figure 1. All subsequent analysis was performed on the Laplacian-transformed EEG signal $\left(\mu V / m^{2}\right)$ computed at each electrode. A subset of 32 electrodes following the standard 10-20 placement was selected for connectivity analysis (Figure 2).

\subsection{Connectivity estimation}

Each recording was segmented into 90 epochs, 6-second-long, relative to the stimulus. Two frequency bands were considered in the analysis: alpha $(8-13 \mathrm{~Hz})$ and beta $(14-30 \mathrm{~Hz})$. Functional connectivity between electrode pairs was computed in several time windows spanning over the epoch. In each frequency band, the window 


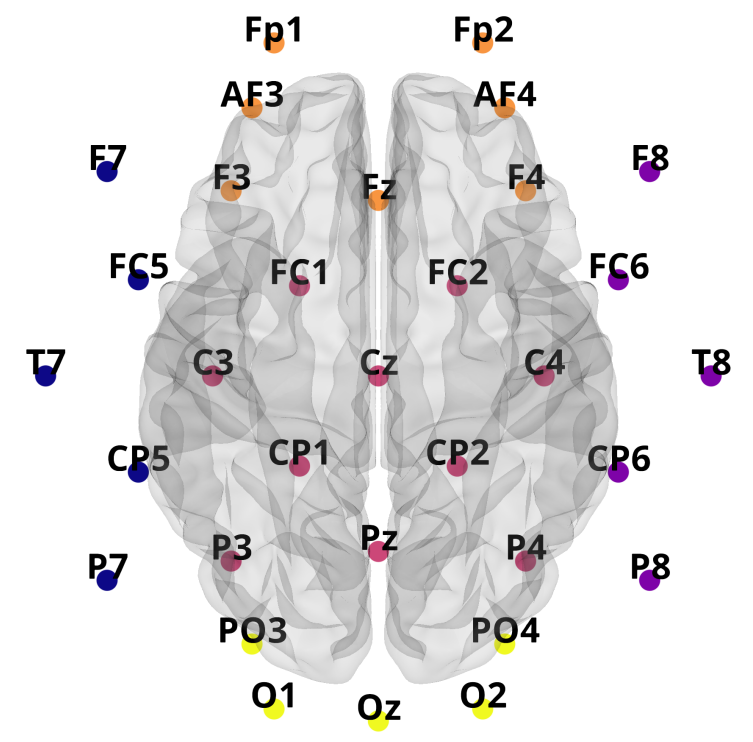

Figure 2: Placement of 32 selected electrodes. To mitigate volume conduction effects, a subset of electrodes was selected to avoid extracting connectivity between spatially close electrodes. The resulting montage had electrodes spaced approximately by $3 \mathrm{~cm}$. All analyses were performed on these 32 electrodes following the standard 10-20 placement. Electrodes were divided into five regions. Two axes were considered for connectivity comparison: lateral (blue-purple) and fronto-occipital (yellow-orange).

length was chosen to contain at least five cycles of the lowest frequency, thus providing a reliable coherency measure [38]. Consequently, a time window of $700 \mathrm{~ms}$ was chosen. In the 4 seconds around the stimulus, 7 time windows at different intervals from the stimulus were analyzed: 6 non-overlapping windows starting at $-2100 \mathrm{~ms}$ ( 3 before stimulus onset, 3 after), and one overlapping window from $-350 \mathrm{~ms}$ to $+350 \mathrm{~ms}$ (centered on stimulus onset). The ImCoh [39], as implemented in MNE, was used as the connectivity measure, and computed within each time window:

$$
\text { connectivity }=\frac{\operatorname{Im}\left(E\left[S_{x y}\right]\right)}{\sqrt{E\left[S_{x x}\right] E\left[S_{y y}\right]}},
$$

where $S_{x y}$ is cross spectral density, $S_{x x}$ and $S_{y y}$ are auto spectral density of EEG signal on electrodes $x$ and $y$, respectively. $E[\cdot]$ denotes an average over epochs.

ImCoh reflects the result of a phase shift between signals, that appear simultaneously on different electrodes. That makes it independent of the volume conduction effects, which often affect EEG-based connectivity, in contrast to the magnitude of the complex value, which is affected by signals with no phase shift. It therefore reflects the true underlying interactions of brain regions; however, some interactions might be lost due to decaying time lag [40]. A method overview is presented in the Figure 3. The connectivity measures were independent of the proximity between electrodes, as no significant relationship between inter-electrode distances and their ImCoh values was observed (Supplementary materials, Figure 2).

Connectivity was computed in the same time window relative to the stimulus, resulting in a symmetrical $32 \times 32$ connectivity matrix, containing ImCoh values, for each subject and time window.

\subsection{Statistical analyses}

Demographic and clinical data were compared between groups using non-parametric two-tailed Mann-Whitney U-test. Statistical analyses of coherence values were restricted to the ImCoh values measured at alpha and beta frequency bands for electrodes grouped into five regions: frontal, central, left and right temporal, and occipital (Figure 3). For each subject, frequency band and condition, at each time window, we computed two summary regional quantities arranged along orthogonal spatial axes of the brain and encompassing distinct functional networks: (1) the average ImCoh value between all pairs of frontal and occipital electrodes (fronto-occipital axis), and (2) the average ImCoh value between all pairs of left and right temporal electrodes (lateral axis). Within each time window, we tested the null hypothesis of no difference between HC and SCZ at the level of these regional summary quantities. Normality of the distribution of average ImCoh in each time window was tested with the Shapiro-Wilk test. Some distributions had p-values below 0.05, showing that they do not fulfill the normality assumption. Therefore, a two-tailed Mann-Whitney U-test with a significance level of 0.05 was used. Within each condition and frequency range, the resulting p-values were then corrected for multiple comparisons across seven-time windows and two region pairs considered, using the Benjamini-Hochberg correction for False Discovery Rate (FDR) control. 


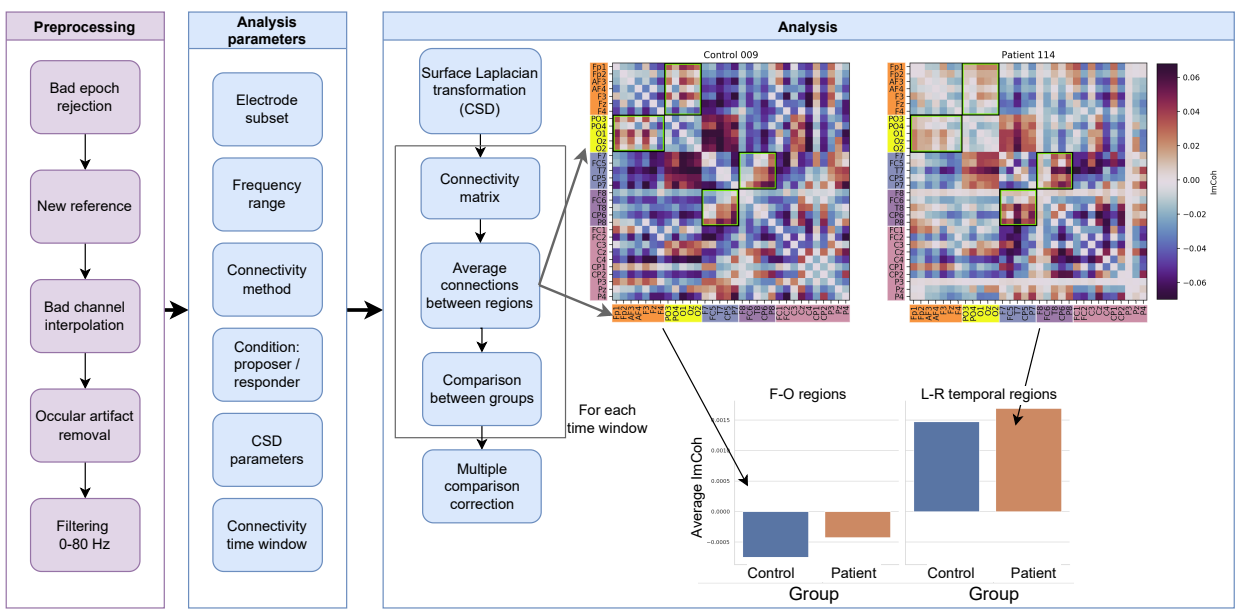

Figure 3: Method overview. The analyses included three treatment blocks: (A) preparation of raw data for subsequent analysis, (B) the estimation of EEG parameters for subsequent connectivity analysis and (C) statistical analysis strategy. Inter-electrode imaginary part of coherency is represented by a matrix for each participant and group (left: one control; right: one patient). Then, regionally-averaged connectivity was compared between patients and controls from these matrices in two different axes: fronto-occipital and lateral (left temporal-right temporal). Results were corrected for multiple comparisons. Histograms refer to the computation of the regional summary quantities. For details see Methods section 2.5

\section{Results}

\subsection{Demographic and clinical data}

The clinical and demographic characteristics of the participants are summarized in Table 1. There were no statistical differences between the two groups on age, sex, and laterality. The scores of the Brief Psychiatric Rating Scale (BPRS) [41], as well as the number of years of education differed significantly. From a neuropsychological point of view, SCZ showed a significant deficit in their performance in all tasks of the Cogstate battery, except for the one-back task. All values are displayed in Table 2.

\begin{tabular}{cccc}
\hline Characteristic & $\begin{array}{c}\text { Patients Group } \\
\text { Mean (SD) }\end{array}$ & $\begin{array}{c}\text { Control Group } \\
\text { Mean (SD) }\end{array}$ & p-value \\
\hline Number of participants & 19 & 24 & - \\
Age (years) & $26.61(4.77)$ & $24.77(3.62)$ & 0.1 \\
Sex (male : female ratio) & $14: 5$ & $9: 15$ & - \\
Laterality (right : left ratio) & $17: 2$ & $20: 4$ & - \\
Education (years) & $12.11(3.37)$ & $15.56(2.79)$ & 0.001 \\
BPRS total & $54.32(9.44)$ & $28.79(2.96)$ & 0.000 \\
BPRS positive symptoms & $12.32(4.08)$ & $4.71(1.06)$ & 0.000 \\
BPRS negative symptoms & $9.89(2.49)$ & $3.08(0.28)$ & 0.000 \\
CPZ equivalents in mg/day & $579.21(554.53)$ & 0 & - \\
\hline
\end{tabular}

Table 1: Demographic and clinical data. BPRS: Brief Psychiatric Rating Scale; CPZ: Chlorpromazine

\subsection{Behavioral data}

Behavioral data including proposition of offers and acceptance rates are presented in Figure 4.

For the proposer condition (Fig. 4 left), the offers made in each group followed a similar distribution, with a majority of offers falling in the middle of the scale. However, SCZ were making on average higher offers than $\mathrm{HC}$, with mean $\mu=5.5$, median $=5$ and standard deviation $\sigma=2.24$. The controls were making on average smaller, and also more consistent offers with $\mu=4.5, \sigma=1.83$, median $=5$. $\left(p=3 * 10^{-44}\right.$, Wilcoxon rank sum test). Regarding the acceptance rates (Fig. 4 right), SCZ were more likely to accept low offers (4 CHF or less), with $30 \%$ average acceptance rate, compared to $17 \%$ for $\mathrm{HC}\left(p=4 * 10^{-7}\right.$, Pearson's Chi-squared Test with continuity correction). At the same time, patients accepted fewer hyperfair (7 CHF or higher), with $89 \%$ of such offers accepted, compared to $99 \%$ hyperfair offers accepted in the controls group $\left(p=2 * 10^{-15}\right.$, Pearson's Chi-squared Test with Yates' continuity correction). These behavioral results are in line with those previously observed in these patients [23]. 


\begin{tabular}{|c|c|c|c|}
\hline & $\begin{array}{l}\text { Controls } \\
\text { Mean (SD) }\end{array}$ & $\begin{array}{l}\text { Patients } \\
\text { Mean (SD) }\end{array}$ & $\mathrm{p}$-value \\
\hline \multicolumn{4}{|l|}{ Executive Function } \\
\hline Set-Shifting Task - ER & $16.2(10.2)$ & $27.9(19.0)$ & 0.0029 \\
\hline \multicolumn{4}{|l|}{ Executive function/spatial problem solving } \\
\hline Groton Maze Learning Test - ER tot & $37.6(7.7)$ & $60.5(25.2)$ & 0.0001 \\
\hline \multicolumn{4}{|l|}{ Psychomotor Function speed of processing } \\
\hline Detection Task speed, $\log 10(\mathrm{~ms})$ & $2.5(0.1)$ & $2.6(0.2)$ & 0.0012 \\
\hline \multicolumn{4}{|l|}{ Visual Attention / Vigilance } \\
\hline Identification Task speed, $\log 10(\mathrm{~ms})$ & $2.7(0.0)$ & $2.7(0.1)$ & 0.0017 \\
\hline \multicolumn{4}{|l|}{ Visual learning and memory } \\
\hline Groton Maze Learning Test - DRE & $3.7(2.7)$ & $8.7(5.9)$ & 0.006 \\
\hline \multicolumn{4}{|l|}{ Verbal learning and memory } \\
\hline \multicolumn{4}{|l|}{ International Shopping List } \\
\hline - CR tot & $30.2(2.6)$ & $26.0(3.9)$ & 0.0002 \\
\hline - DR & $10.8(1.3)$ & $8.6(1.9)$ & 0.0001 \\
\hline \multicolumn{4}{|l|}{ Working Memory } \\
\hline One Back Task (Acc) & $1.3(0.2)$ & $1.2(0.2)$ & 0.074 \\
\hline \multicolumn{4}{|l|}{ Social Cognition } \\
\hline Social-Emotional Cognition Task (Acc) & $1.2(0.1)$ & $1.0(0.2)$ & 0.002 \\
\hline
\end{tabular}

Table 2: Neuropsychological tasks scores. Data are presented as mean (SD). Acc, accuracy of performance (arcsine transformation of the square root of the proportion of correct responses); ER tot, total number of errors; DRE, delayed recall (number of correct responses); CR tot, total number of correct responses. See text for details. Significant differences were found for each test except the one back task
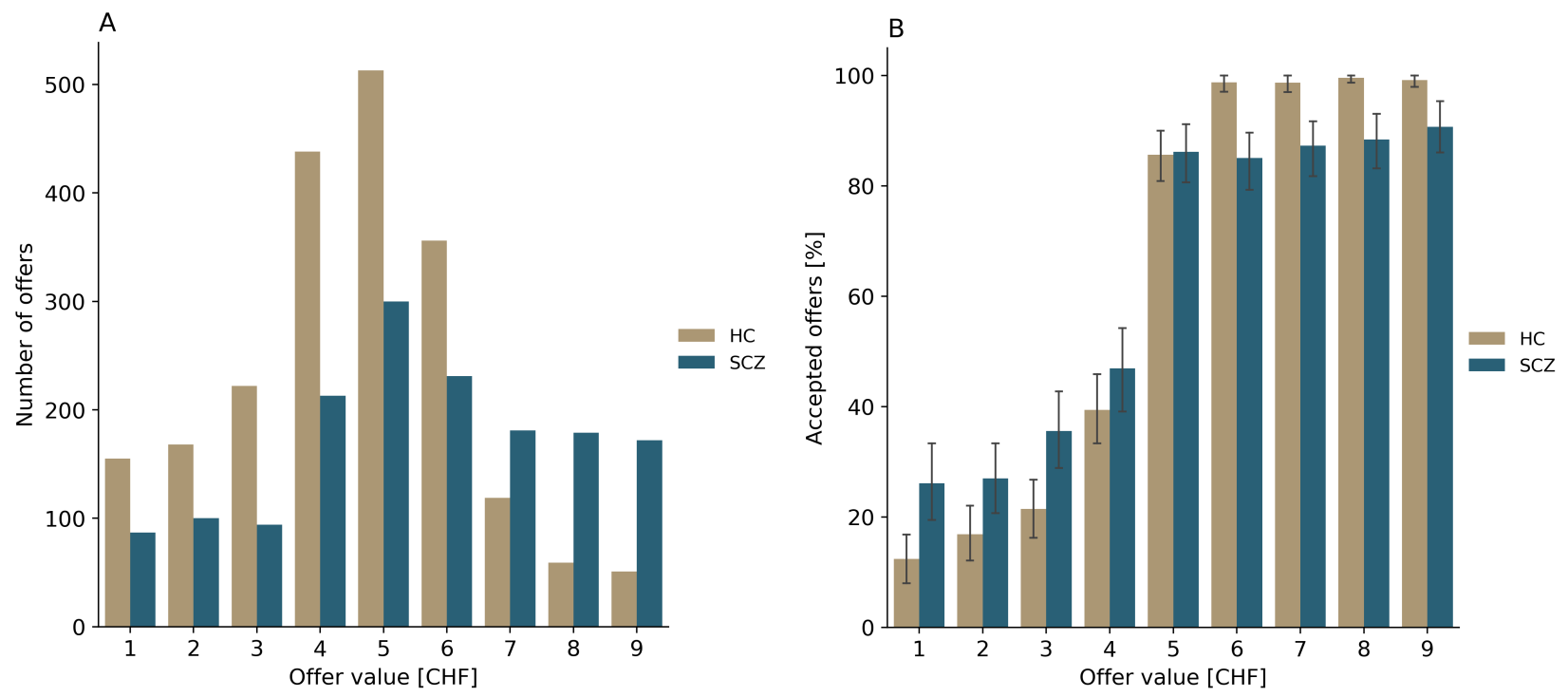

Figure 4: Behavioural scores for healthy controls and patients.

A: Distribution of the proposed offers. Number of all offers in the group made by the healthy controls (brown; mean $=4.5 \pm 1.83$ ) and the patients with schizophrenia (blue; mean $=5.5 \pm 2.24$ ) groups when acting as proposers.

B: Acceptance rates of each offers. Acceptance rates of healthy controls (brown) and patients with schizophrenia (blue) groups when acting as responders. The bars represent the percentage of accepted offers for each amount with standard deviations $( \pm \mathrm{SD})$. Significant differences were found for the acceptance rates between the two groups. 


\subsection{Dynamic functional connectivity}

The imaginary part of coherency (ImCoh) for both frequency bands of interest was computed to investigate whether the functional connectivity differed between groups in the proposer and responder conditions. Average ImCoh in both groups in proposer $(\mathrm{A})$ and responder $(\mathrm{B})$ conditions in alpha $(8-13 \mathrm{~Hz})$ and beta (14-30 $\mathrm{Hz})$ bands are illustrated in Figures 5 and 7, respectively. In the alpha $(8-13 \mathrm{~Hz})$ band (Fig. 5a), there was no difference between groups at any time relative to the stimulus in the proposer condition. In contrast, there was a significant difference in fronto-occipital connectivity between patients and controls $\left(p_{B H}=0.043\right.$, corrected for multiple comparisons using Benjamini-Hochberg procedure) during the interval -0.7 to 0 sec before stimulus onset in responder condition (Fig. 5b). The distribution of fronto-occipital ImCoh values in this interval is presented in Figure6. No significant difference was observed in any of the other time windows.

No difference of connectivity between symmetrical temporal regions was observed in the beta (14-30 Hz) band between groups in both proposer (Fig. 7a) and responder (Fig. 7b) conditions in any time window, from -2 sec to 2 seconds post-stimulus.

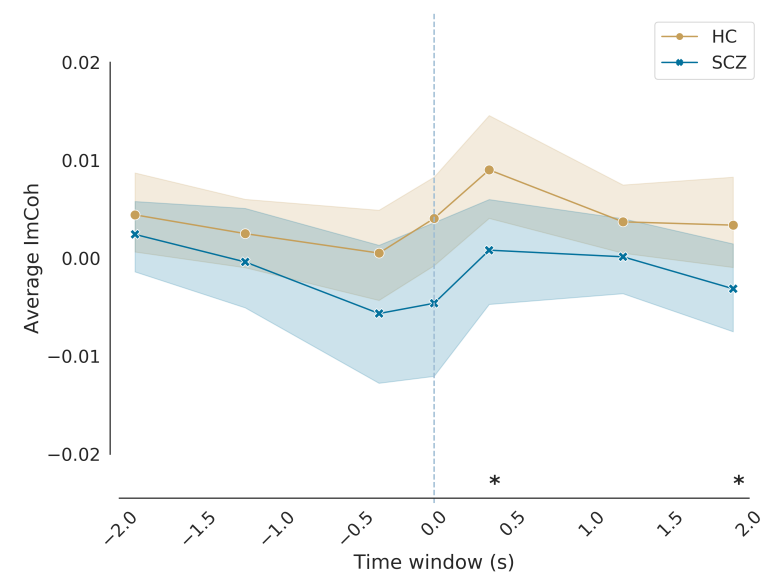

(a) Proposer condition, $8-13 \mathrm{~Hz}$

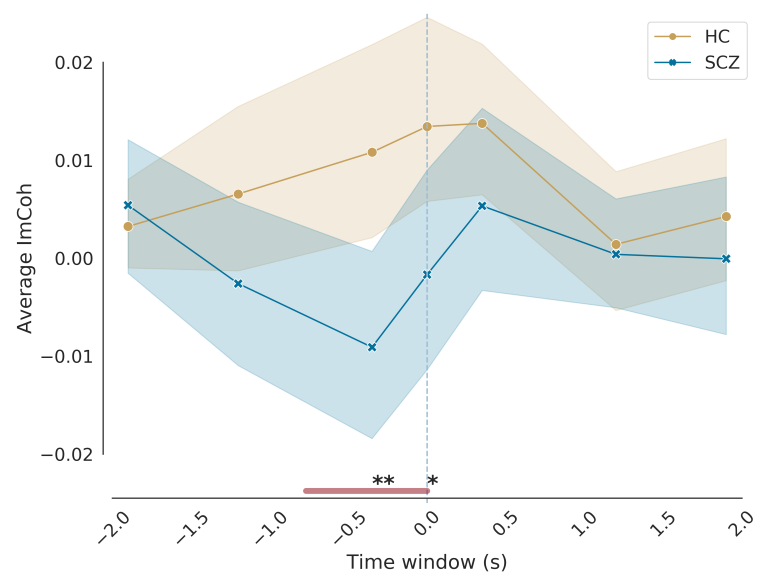

(b) Responder condition, $8-13 \mathrm{~Hz}$

Figure 5: Average connectivity between frontal and occipital electrodes in the alpha (8-13 Hz) band in proposer (A) and responder (B) conditions in a $2 \mathrm{~s}$ lag time centered on the stimulus onset. Vertical line at 0s shows the stimulus onset. Line markers indicate the centers of analysis windows. Note the significant difference between groups in the responder condition in a time window of $0.7 \mathrm{~s}$ before stimulus onset; ** Mann-Whitney test p-value $<0.05$ (corrected for multiple comparisons by Benjamini-Hochberg procedure), ${ }^{*}$ p-value $<0.05$ (uncorrected). No significant differences in connectivity strength between groups were observed in the proposer condition. See results section for details. 

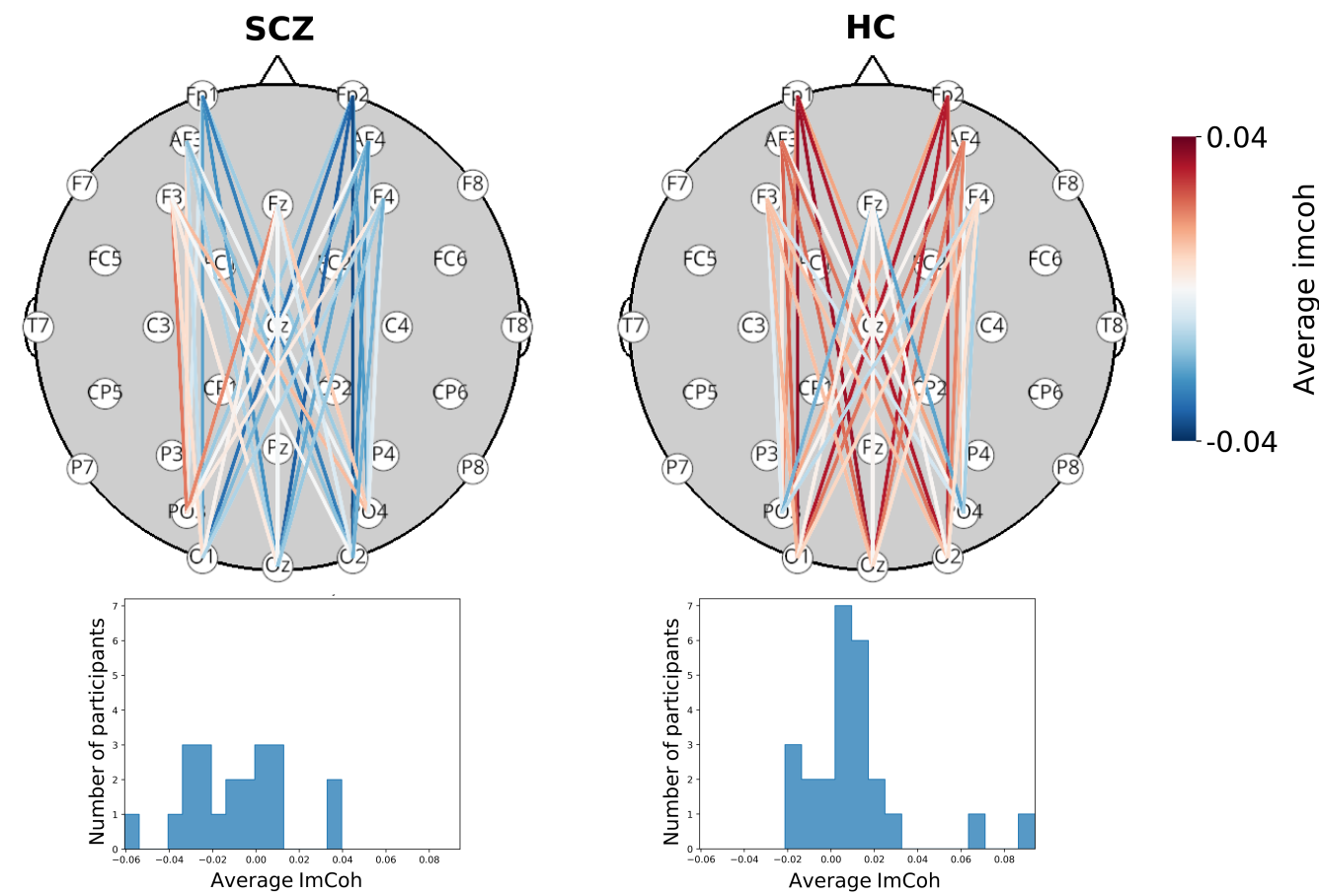

Figure 6: Top: Proposer condition effect on fronto-occipital electrodes pairs coherence in 8-13Hz frequency band during the time window $-0.7 \mathrm{~s}$ to $0 \mathrm{~s}$ before the stimulus onset. Values of imaginary part of coherency for each fronto-occipital electrode pair were averaged across all subjects of SCZ (A1) and HC (B1) groups. Bottom: Subject-level distribution of averaged fronto-occipital connectivity values in SCZ (A2) and HC (B2) groups in the time interval centered at $-0.35 \mathrm{~s}$. The distribution of imaginary part of coherency values was negative for SCZ $(-0.009 \pm 0.023)$ but positive for HC $(0.011 \pm 0.024)$ group. Note the shift towards negative values visible in schizophrenia patients.

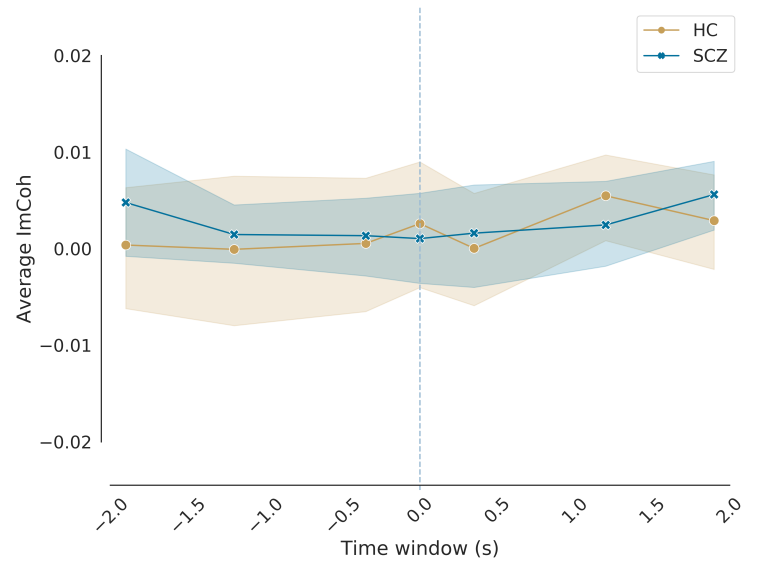

(a) Proposer condition, $14-30 \mathrm{~Hz}$

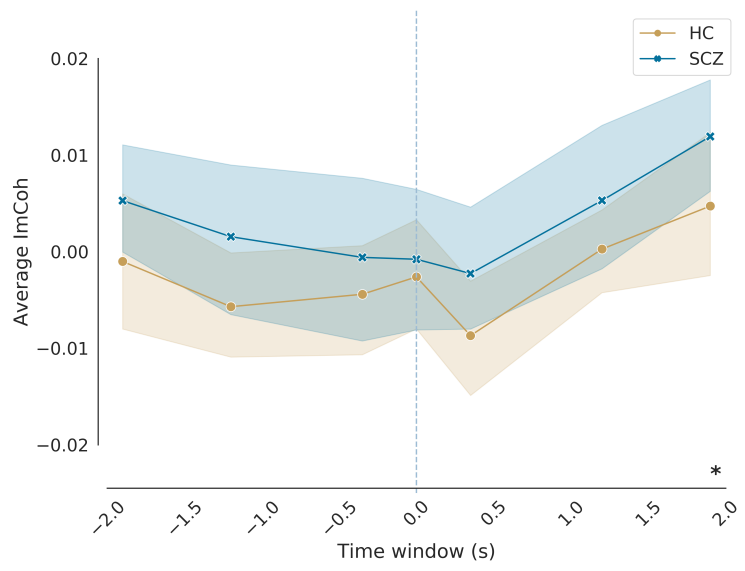

(b) Responder condition, $14-30 \mathrm{~Hz}$

Figure 7: Average connectivity between left and right temporal electrodes for the beta (14-30 $\mathrm{Hz})$ band in proposer (A) and responder (B) conditions, in a $2 \mathrm{~s}$ lag time centered on the stimulus onset. Vertical line at Os shows the stimulus onset. Note that no significant difference between groups was observed in proposer and responder conditions after correcting for multiple comparisons. 


\section{Discussion}

We previously reported disturbance of a late ERP component associated with difficulties in interpreting another's behavior in schizophrenia patients [23]. The present work was designed to further study the nature of this disrupted neurophysiology in schizophrenia. In agreement with the hypothesis that transient synchronization of oscillations between brain structures largely support the cognitive functions [42], we observed significant differences in fronto-occipital connectivity in patients with schizophrenia. These impairments of connectivity appear strictly independent of top-down attention and decision making, since they were neither found for inter-temporal connectivity in the beta band, nor after relevant stimulus onset. The aberrant network activity of oscillations in the alpha band clearly demonstrates a deficit in anticipating the behavior of others during interaction with the opposite player for final decision making. In addition, the difference was related to the responder condition, arguing in favor of a deficit between the two UG conditions that depends on the symbolic position of the player.

Neuronal oscillations are the fundamental mechanism for establishing a precise temporal relationship between different brain areas, and underlie high-level cognitive processes [43]. EEG/MEG studies support the idea that phase coherence for a specific frequency provides an excellent method to study brain interactions [44] by establishing a specific temporal relationship between different brain areas. In agreement with this hypothesis, we thus used the imaginary part of coherency, corresponding to the phase in Cartesian representation, to analyze brain interactions. This method allowed us to show significant differences in a frequency band between HC and SCZ before relevant stimulus onset in the responder condition. The observed changes in the brain network suggest a disturbance of the local cortical excitability transmission, which would then lead to disruption of the cognitive alpha band-related functions in patients. Physiologically, alpha activity coordinates inter-regional impulses, resulting in an active increase in cortical information processing under normal condition [39]. In this context, our results are consistent with a disruption in temporal communication between brain regions associated with cognitive states in SCZ patients [43] preceding the early stages of information processing and yield additional support to the presence of a functional disconnection disorder in this population [45].

Our findings of a significant difference of connectivity between fronto-occipital regions in the alpha band are consistent with the topology of the network involved in social cognition [46]. This refers to introduction of steps leading to anticipate the counterpart's emotion and intentions of other's in an environment of rapid social interaction for subsequent decision making.

Previous physiological studies revealed that the co-activation of frontal and occipital alpha-synchronized areas would allow fast identification in a decision situation for subsequent choices and facilitate processes of an anticipatory nature. In line with this first hypothesis, a recent study based on multisensory perception revealed that a regulation of perceptual gains in attentional conditions was exerted by frontal alpha power oscillations, supporting the view of top-down attentional control to sensory cortices via alpha oscillations in attention-dependent conditions [47]. Meanwhile, alpha oscillations in the occipital area are strongly involved in the processing of visual stimuli [48, 49]. Cognitively, a decrease in alpha amplitude in the occipital area [50] was described during anticipation of predictive contextual images, highlighting that this cognitive process is tightly alpha-related. Further studies have confirmed these results [17, 27]. Most importantly, a rhythmic increase of alpha amplitude was reported right before the expected reappearance of a target in a perceptual discrimination task [51], proving that the modulation of the alpha rhythm reflects the pre-activation of cortical areas involved in the processing of the decisive stimulus and consequently facilitating the anticipation process. In the present study, significant differences were observed in the lag time directly coinciding with the expectation or anticipation of the target interactive stimulus. Therefore, our findings strongly support to alteration of phase coherency between occipital and frontal regions due to a disruption of cortical excitability in schizophrenic patients [52], thereby impairing mentalization abilities that occur very shortly before social interaction with a second person.

In line with our results, these two brain regions are functionally activated in social games and in the tasks of assigning mental states to oneself and others, also known as the theory of the mind (ToM) which is a domain where schizophrenic patients have disabilities. Previous research in controls even demonstrated that the strength of fronto-occipital alpha oscillations synchronization in the resting-state EEG was significantly correlated with the performance in the UG task [18]. The present functional abnormalities may reflect a poor integration of information from their partner, which is suggested by the statistical deviation between acceptance of fair and unfair propositions in the responder condition in the SCZ patient. In line with our previous results [23], SCZ patients exhibit problems in understanding the reasoning behind offers made by the other player (responder condition). In contrast, no physiological difference was observed between the two groups in the proposer condition, suggesting that patients with schizophrenia playing as proposers are able to anticipate and adaptively simulate the emotions, both deliberative (accepted) and affective (rejected) motives, of the second player. Yet, SCZ proposed higher offers than HC, which partly disagrees with this result. Medial prefrontal alpha and beta oscillations decrease modulation during anticipation of the other's response was previously reported in patients with schizophrenia [22], contrasting with the present findings. Two main methodological differences may explain this discrepancy. First, the time-frequency method which computes significant frequency deviations from baseline random fluctuations after stimulus onset was used in this previous work and was thus not adapted 
to compute oscillations synchronization, whilst we investigated functional connectivity between brain regions. Second, signal measurements in [22] could suffer from surface conduction effects due to proximity, which is not the case in our study.

On the other hand, no inter-temporal connectivity in beta band was observed between the groups in both conditions. This result disagrees with the conclusions of Park et al. [53] who reported that the ability to infer judgments of others was correlated to beta power rTPJ in the healthy population. In contrast, injuries are mainly lateralized in the left temporal lobe in schizophrenia.

Several limitations should be considered when interpreting the present data. First, while we controlled for demographic factors, drug intake and patients were as cognitively homogeneous as possible, clinical heterogeneity of the disease might have an impact on the data. Second, we opted for the reciprocal interaction between the participant and the interactive stimulus acting as responder (single-brain approach). Subsequently, our social interaction paradigm is not optimal to detect additional synchronized activation probably engaged during a social interaction between two individuals (simultaneous dual-brain approach). Future activation studies using simultaneous sequential dual-brain approach and the combination of EEG recordings with other functional imaging modalities, as well as fully reciprocal, face-to-face interactions with a real social partner are required to further explore the mentalizing network alterations associated with schizophrenia. Lastly, the limited sample size imposed restrictions on the spatial specificity of the findings, meaning that we could not investigate connectionlevel differences between groups, and instead had to summarize results into broad spatial patterns. To overcome this, future work could either restrict temporal specificity, or increase subject recruitment.

\section{Conclusions}

Overall, our results for the alpha band range support the presence of a disruption of cortical functional connectivity involved in mechanisms of social interaction in the responder condition, during anticipation of the offer. Conversly, our results suggest that mentalization abilities engaged before the relevant stimulus onset were not altered in patients with schizophrenia in the proposer condition.

\section{Data availability}

Data used in this paper are available under a CC0 license at https://openneuro.org/datasets/ds004000. Code to reproduce analyses is available as open source at https://c4science.ch/source/EEG_conn/.

\section{Acknowledgments}

The authors gratefully acknowledge the psychiatrists of the Mental Health Network Fribourg (RFSM) for the diagnosis and recruitment of patients. We also thank Gijs Plomp for his helpful input on electrode-space connectivity.

\section{Author Contributions}

A. Padée and J. Richiardi contributed to analysis and interpretation of data, and to drafting and revising the manuscript. P. Missonnier contributed to the study conception and design, interpretation of data, to drafting and revising the manuscript. G. Favre acquired the EEG and neuropsychological data. I. Gothuey contributed to the recruitment of patients, characterization and design of the study, and to revising the manuscript. A. Prévot contributed to drafting and revising the manuscript. All authors approved the final version of the manuscript.

\section{Competing interests' statement}

The authors declare no competing interests.

\section{References}

1. American Psychiatric Association. Diagnostic and Statistical Manual of Mental Disorders Fifth Edition. https : //psychiatryonline .org/doi/book/10.1176/appi .books. 9780890425596 (2021) (American Psychiatric Association, May 22, 2013).

2. Tan, B.-L., Lee, S.-A. \& Lee, J. Social cognitive interventions for people with schizophrenia: A systematic review. Asian Journal of Psychiatry 35, 115-131. ISSN: 1876-2026 (June 2018). 
3. Gottesman, I. I. \& Gould, T. D. The endophenotype concept in psychiatry: etymology and strategic intentions. The American Journal of Psychiatry 160, 636-645. ISSN: 0002-953X (Apr. 2003).

4. Braff, D. L., Freedman, R., Schork, N. J. \& Gottesman, I. I. Deconstructing schizophrenia: an overview of the use of endophenotypes in order to understand a complex disorder. Schizophrenia Bulletin 33, 21-32. ISSN: 0586-7614 (Jan. 2007).

5. Kirk, U., Downar, J. \& Montague, P. R. Interoception drives increased rational decision-making in meditators playing the ultimatum game. Frontiers in Neuroscience 5, 49. ISSN: 1662-453X (2011).

6. Wang, Y. et al. Ingroup/outgroup membership modulates fairness consideration: neural signatures from ERPs and EEG oscillations. Scientific Reports 7, 39827. ISSN: 2045-2322 (Jan. 4, 2017).

7. Rubinstein, A. Perfect Equilibrium in a Bargaining Model (1982).

8. Schuster, S. A New Solution Concept for the Ultimatum Game leading to the Golden Ratio. Scientific Reports 7, 5642. ISSN: 2045-2322 (July 17, 2017).

9. Billeke, P. \& Aboitiz, F. Social cognition in schizophrenia: from social stimuli processing to social engagement. Frontiers in Psychiatry 4, 4. ISSN: 1664-0640 (2013).

10. Van 't Wout, M. \& Sanfey, A. G. Interactive decision-making in people with schizotypal traits: a game theory approach. Psychiatry Research 185, 92-96. ISSN: 0165-1781 (Jan. 30, 2011).

11. Wischniewski, J. \& Brüne, M. Moral reasoning in schizophrenia: an explorative study into economic decision making. Cognitive Neuropsychiatry 16, 348-363. ISSN: 1464-0619 (2011).

12. De la Asuncion, J., Docx, L., Sabbe, B., Morrens, M. \& de Bruijn, E. R. A. Abnormal emotion processing, but intact fairness and intentionality considerations during social decision-making in schizophrenia. Frontiers in Psychology 6, 1058. ISSN: 1664-1078 (2015).

13. Dodell-Feder, D., Tully, L. M., Lincoln, S. H. \& Hooker, C. I. The neural basis of theory of mind and its relationship to social functioning and social anhedonia in individuals with schizophrenia. NeuroImage. Clinical 4, 154-163. ISSN: 2213-1582 (2014).

14. Fell, J. \& Axmacher, N. The role of phase synchronization in memory processes. Nature Reviews. Neuroscience 12, 105-118. ISSN: 1471-0048 (Feb. 2011).

15. Nácher, V., Ledberg, A., Deco, G. \& Romo, R. Coherent delta-band oscillations between cortical areas correlate with decision making. Proceedings of the National Academy of Sciences of the United States of America 110, 15085-15090. ISSN: 1091-6490 (Sept. 10, 2013).

16. Battaglia, F. P. \& McNaughton, B. L. Polyrhythms of the brain. Neuron 72, 6-8. ISSN: 1097-4199 (Oct. 6, 2011).

17. Simonet, M. et al. The modulation of event-related alpha rhythm during the time course of anticipation. Scientific Reports 9. Bandiera_abtest: a Cc_license_type: cc_by Cg_type: Nature Research Journals Number: 1 Primary_atype: Research Publisher: Nature Publishing Group Subject_term: Cognitive neuroscience;Neuroscience Subject_term_id: cognitive-neuroscience;neuroscience, 18226. ISSN: 2045-2322. https : //www.nature.com/articles/s41598-019-54763-1 (2021) (Dec. 3, 2019).

18. Si, Y. et al. Predicting individual decision-making responses based on the functional connectivity of restingstate EEG. Journal of Neural Engineering 16, 066025. ISSN: 1741-2552 (2019).

19. Palmer, C., Zapparoli, L. \& Kilner, J. M. A New Framework to Explain Sensorimotor Beta Oscillations. Trends in Cognitive Sciences 20. Number: 5, 321-323. ISSN: 1879-307X (May 2016).

20. Krall, S. C. et al. The role of the right temporoparietal junction in attention and social interaction as revealed by ALE meta-analysis. Brain Structure 6 Function 220, 587-604. ISSN: 1863-2661 (Mar. 2015).

21. Wang, X.-J. Neurophysiological and computational principles of cortical rhythms in cognition. Physiological Reviews 90, 1195-1268. ISSN: 1522-1210 (July 2010).

22. Billeke, P. et al. Paradoxical Expectation: Oscillatory Brain Activity Reveals Social Interaction Impairment in Schizophrenia. Biological Psychiatry 78, 421-431. ISSN: 1873-2402 (Sept. 15, 2015).

23. Horat, S. K. et al. Impaired social cognition in schizophrenia during the Ultimatum Game: An EEG study. Schizophrenia Research 192, 308-316. ISSN: 0920-9964. https : //www . sciencedirect . com/science/ article/pii/S0920996417303110 (2021) (Feb. 1, 2018).

24. WHO. The ICD-10 classification of mental and behavioural disorders : diagnostic criteria for research English, xiii, 248 p. ISBN: 9241544554 (World Health Organization Geneva, 1993).

25. Ventura, J. et al. Brief Psychiatric Rating Scale Expanded version 4.0: Scales anchor points and administration manual. Int J Meth Psychiatr Res 13, 221-244 (Jan. 1, 1993).

26. Sanfey, A. G., Rilling, J. K., Aronson, J. A., Nystrom, L. E. \& Cohen, J. D. The Neural Basis of Economic Decision-Making in the Ultimatum Game. Science 300. Publisher: American Association for the Advancement of Science Section: Report, 1755-1758. ISSN: 0036-8075, 1095-9203. https://science.sciencemag. org/content/300/5626/1755 (2020) (June 13, 2003). 
27. Radke, S., Guroglu, B. \& de Bruijn, E. There's Something about a Fair Split: Intentionality Moderates Context-Based Fairness Considerations in Social Decision-Making. PloS one 7, e31491 (Feb. 17, 2012).

28. Jung, T. P. et al. Removing electroencephalographic artifacts by blind source separation. Psychophysiology 37, 163-178. ISSN: 0048-5772 (Mar. 2000).

29. Klados, M. A., Papadelis, C., Lithari, C. D. \& Bamidis, P. D. The Removal Of Ocular Artifacts From EEG Signals: A Comparison of Performances For Different Methods in 4th European Conference of the International Federation for Medical and Biological Engineering (eds Vander Sloten, J., Verdonck, P., Nyssen, M. \& Haueisen, J.) (Springer, Berlin, Heidelberg, 2009), 1259-1263. ISBN: 978-3-540-89208-3.

30. Perrin, F., Bertrand, O. \& Pernier, J. Scalp current density mapping: value and estimation from potential data. IEEE transactions on bio-medical engineering 34, 283-288. ISSN: 0018-9294 (Apr. 1987).

31. Babiloni, F. et al. Spline Laplacian estimate of EEG potentials over a realistic magnetic resonanceconstructed scalp surface model. Electroencephalography and Clinical Neurophysiology 98, 363-373. ISSN: 0013-4694 (Apr. 1996).

32. Srinivasan, R., Winter, W. R., Ding, J. \& Nunez, P. L. EEG and MEG coherence: measures of functional connectivity at distinct spatial scales of neocortical dynamics. Journal of neuroscience methods 166, 4152. ISSN: 0165-0270. https : //www.ncbi.nlm.nih.gov/pmc/articles/PMC2151962/ (2021) (Oct. 15, 2007).

33. Kayser, J. \& Tenke, C. E. On the benefits of using surface Laplacian (current source density) methodology in electrophysiology. International Journal of Psychophysiology. On the benefits of using surface Laplacian (current source density) methodology in electrophysiology 97, 171-173. ISSN: 0167-8760. https : //www . sciencedirect.com/science/article/pii/S0167876015002111 (2021) (Sept. 1, 2015).

34. Nunez, P. L. et al. A theoretical and experimental study of high resolution EEG based on surface Laplacians and cortical imaging. Electroencephalography and Clinical Neurophysiology 90, 40-57. ISSN: 0013-4694 (Jan. 1994).

35. Nunez, P. L. \& Srinivasan, R. A theoretical basis for standing and traveling brain waves measured with human EEG with implications for an integrated consciousness. Clinical Neurophysiology: Official Journal of the International Federation of Clinical Neurophysiology 117, 2424-2435. ISSN: 1388-2457 (Nov. 2006).

36. Tenke, C. E. \& Kayser, J. Reference-free quantification of EEG spectra: combining current source density (CSD) and frequency principal components analysis (fPCA). Clinical Neurophysiology: Official Journal of the International Federation of Clinical Neurophysiology 116, 2826-2846. ISSN: 1388-2457 (Dec. 2005).

37. Gramfort, A. et al. MEG and EEG data analysis with MNE-Python. Frontiers in Neuroscience 7. ISSN: 1662-453X. https://www.frontiersin.org/articles/10.3389/fnins.2013.00267 (2022) (2013).

38. Leonardi, N. \& Van De Ville, D. On spurious and real fluctuations of dynamic functional connectivity during rest. NeuroImage 104, 430-436. ISSN: 1053-8119. http : / / www . sciencedirect . com/science / article/pii/S1053811914007496 (2020) (Jan. 1, 2015).

39. Bowyer, S. M. Coherence a measure of the brain networks: past and present. Neuropsychiatric Electrophysiology 2, 1. ISSN: 2055-4788. https : //doi .org/10.1186/s40810-015-0015-7 (2021) (Jan. 17, 2016).

40. Nolte, G. et al. Identifying true brain interaction from EEG data using the imaginary part of coherency. Clinical Neurophysiology: Official Journal of the International Federation of Clinical Neurophysiology 115, 2292-2307. ISSN: 1388-2457 (Oct. 2004).

41. Overall, J. E. \& Gorham, D. R. The Brief Psychiatric Rating Scale. Psychological Reports 10. Publisher: SAGE Publications Inc, 799-812. ISSN: 0033-2941. https : //doi .org/10 . 2466/pr0 .1962 .10.3.799 (2022) (June 1, 1962).

42. Buzsáki, G. \& Wang, X.-J. Mechanisms of gamma oscillations. Annual Review of Neuroscience 35, 203225. ISSN: 1545-4126 (2012).

43. Hunt, M. J., Kopell, N. J., Traub, R. D. \& Whittington, M. A. Aberrant Network Activity in Schizophrenia. Trends in Neurosciences 40, 371-382. ISSN: 1878-108X (June 2017).

44. Tononi, G., Edelman, G. M. \& Sporns, O. Complexity and coherency: integrating information in the brain. Trends in Cognitive Sciences 2, 474-484. ISSN: 1364-6613 (Dec. 1, 1998).

45. Friston, K., Brown, H. R., Siemerkus, J. \& Stephan, K. E. The dysconnection hypothesis (2016). Schizophrenia Research 176, 83-94. ISSN: 1573-2509 (Oct. 2016).

46. Green, M. F., Horan, W. P. \& Lee, J. Social cognition in schizophrenia. Nature Reviews. Neuroscience 16, 620-631. ISSN: 1471-0048 (Oct. 2015). 
47. Misselhorn, J., Friese, U. \& Engel, A. K. Frontal and parietal alpha oscillations reflect attentional modulation of cross-modal matching. Scientific Reports 9. Bandiera_abtest: a Cc_license_type: cc_by Cg_type: Nature Research Journals Number: 1 Primary_atype: Research Publisher: Nature Publishing Group Subject_term: Attention;Human behaviour Subject_term_id: attention;human-behaviour, 5030. ISSN: 20452322. https://www.nature.com/articles/s41598-019-41636-w (2021) (Mar. 22, 2019).

48. Klimesch, W., Sauseng, P. \& Hanslmayr, S. EEG alpha oscillations: the inhibition-timing hypothesis. Brain Research Reviews 53. Number: 1, 63-88. ISSN: 0165-0173 (Jan. 2007).

49. Klimesch, W. Alpha-band oscillations, attention, and controlled access to stored information. Trends in Cognitive Sciences 16, 606-617. ISSN: 1364-6613. https://www.sciencedirect.com/science/article/ pii/S1364661312002434 (2021) (Dec. 1, 2012).

50. Bidet-Caulet, A. et al. Dynamics of anticipatory mechanisms during predictive context processing. The European journal of neuroscience 36, 2996-3004. ISSN: 0953-816X. https://www.ncbi.nlm.nih.gov/ pmc/articles/PMC3463677/ (2021) (Oct. 2012).

51. Rohenkohl, G. \& Nobre, A. C. Alpha Oscillations Related to Anticipatory Attention Follow Temporal Expectations. Journal of Neuroscience 31. Number: 40 Publisher: Society for Neuroscience Section: Articles, 14076-14084. ISSN: 0270-6474, 1529-2401. https ://www.jneurosci.org/content/31/40/14076 (2021) (Oct. 5, 2011).

52. Roopun, A. K. et al. Region-Specific Changes in Gamma and Beta2 Rhythms in NMDA Receptor Dysfunction Models of Schizophrenia. Schizophrenia Bulletin 34, 962-973. ISSN: 0586-7614. https : //www . ncbi.nlm.nih.gov/pmc/articles/PMC2518640/ (2022) (Sept. 2008).

53. Park, J., Kim, H., Sohn, J.-W., Choi, J.-r. \& Kim, S.-P. EEG Beta Oscillations in the Temporoparietal Area Related to the Accuracy in Estimating Others' Preference. Frontiers in Human Neuroscience $12,43$. ISSN: 1662-5161. https://www.frontiersin.org/article/10.3389/fnhum. 2018.00043 (2021) (2018). 